


$$
\text { S62 }
$$



f.11668 
Digitized by the Internet Archive. in 2016 with funding from Getty Research Institute 
2 mogr 2208 
C A T A L O G U E DES BRONZES

\section{ET AUTRES CURIOSITES}

Egyptiennes, Etrufques, Indiennes \& Chinoifes; Médailles \& Monnoies d'or \& d'argent ; Bagues \&2 Boutons de diamants ; Pierres gravées, Agates, Jafpes \& Coquilles, dont la Scalata; Po. lypiers, Animaux, Oifeaux \& Infectes: Pieces anatomiques, \& nombres d'autres morcenux curieux.

$D u$ Cabinet de feu M. MORAND, Chevalier de l'Ordre du Roi, Infpecteur, Fenfionnaire de l'Académie Royale des Ghisurgig fcionos Membre de la Société Royale de Londres, des Académies de Bologne, Si. Petersbourg, de Porto, Ec.

Cette vente fe fera le Mercredi premier DGcemoi es,rois heures de relevée, \& jours fuivants, tue de Grenelle, près celle des Saints Pères.
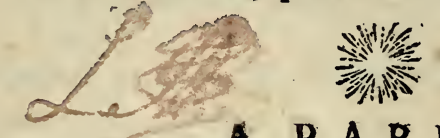

\section{A PARIS,}

$\left\{\begin{array}{l}\text { R E M Y, rue des grands Augultíns; } \\ \text { près la rue Chriftine. }\end{array}\right.$

Chez $\{$ près la rue Chriftine. MU S IER, Pere, Libraire, quaì des Augurtins.

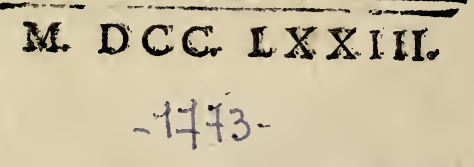





\section{T A B L E}

Bronzes $\&$ autres antiquités. page I

Figures Egyptiennes.

Bronzes Indiens \& Chinois.

Différens Bronzes.

$V$ afes antiques de verre.

Bas-reliefs, Buftes de marbre, Terre cuite, \&c.

Figures des Indes.

Tableaux mouvans, \&zc.

Médailles \& Monnoies d'or , d'argent, $\&$ de cuivre. Armes, Uftenfiles, \& Habillemens Indiens \& Chinois.

Laques \& Ivoire.

24

26

Mines d'or, d'argent $\&$ autres. $\quad 27$ Subftances falines \& inflammables. 22 Cryftaux \& Cryftallifations, Bagues de diamants, dont une de conféquence.

idem

Pierres gravées $\&$ autres montées en Bagues.

Boutons de diamants.

Pierres fines non montées.

Agates, Jafpes , \&zc. 30

Coquilles univalves $\&$ bivalves. 36 . 


\section{T A B L E.}

Polypiers.

Animaux, Oifaux \& 40

Animaux, Oifeaux \&Infectes. $4 I$

Cabinet anatomique.

Pieces en ivoire.

Pieces en bois $\&$ en cryftal.

Pieces de Méchanique.

45

Différens Bijoux, \& autres effets. $\sigma_{2}$ 


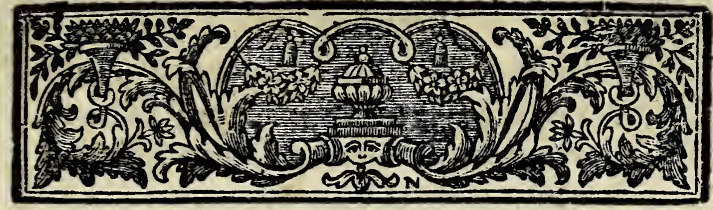

\section{A T A L O G UE}

Des Objets curieux du Cabinet de feu M. MO RA N D, Chevalier de l'OrdredeS. Michel.

BRONZES, E autres ANTIQUITÉS.

$1 D_{\text {E } ~} \times$ différentes figures de $\mathrm{J}_{\mathrm{U}}$ piter, l'une porte 4 pouces 3 lignes de hauteur, l'autre 3 pouces $9 \mathrm{di}-$ gnes, fur des pieds de bois.

2 La ftatue de Jupiter \& celle de Mercure, l'une \& l'autre mutilées; ces bronzes portent chaque 4 pouces 3 lignes, non compris des pieds de $s-1$. bois noirci.

3 Mercure affis, avec les taloniaieres \& le pétafe ou chapeau âlé: ce joli 16.2. bronze porte 4 pouces 6 lignes.

4 Deux figures de Mercure tenant la 12, 5 A 
bourfe, l'un affis, hauteur 5 pouces, fur un pied de marbre ; l'autre cebout, hauteur 4 pouces 6 lignes, fur un focle de bois noirci.

9 is Statue de Mercure tenant labourfe, hauteur de 6 pouces 6 lignes, fur un pied de marqueterie à filets de

9. 66 Le Mercure de Jean de Bologne; ce bronze élégant porte 6 pouces 3 lignes de haur.

J - 1. 7 Deux figures de Vénus, l'une drapée, l'autre nue, hauteur de chacune 4 pouces 3 lignes, fur des pieds douches de bois noirci.

4. 15.8 Une petite figure de femme nue, afiríe la main polée fur un carquois, hauteur 3 pouces, fur un pied de bois noirci.

7 - 10. 9 Jeune homme portant fes mains à la tête, figure inconnue moulée en cire perdue, hauteur 6 pouces $6 \mathrm{li}$.

$g$. 5 Vónus nue tenant la pomme; ce joli bronze portes pouces de haur. 9. 7 I I Vénus pudique, hauteur 5 pouces 9 lignes.

12 Heicule tenant fa malfue fous fon

$g$ - ig . bras gauche, hauteur 9 pou. 6 lig. 


\section{Bronz̧es.}

13 Trois figures d'Hercule, dont deux avec la peau de lion, l'autre avec Cerbere : ce dernier porte 4 pouces de hauteur.

14 Deux autres Hercules avec la peau. de lion, l'un de 5 pouces 3 lignes, $12-1$. fes pieds font mutilés; l'autre dans le goût Etrufque, hauteur 3 pouces 6 lignes, fur un pied d'albâtre.

is Une figure d'Apollon, hauteur 5 pouces, \& une de Pallas de 3 pouces 3 lignes.

16 Une Pallas, figure antique fur fon pied de bronze, hauteur totale 10 pouces 9 lignes.

17 Autre figure de Pallas, hauteur 6 6. Iy. pouces, fur un pied de bois.

18 Le Dieu Mars, figure en pied de. 10 pouces 6 lignes de hauteur.

19. Une figure d'Atlas d'un bon goût ; hauteur 4 pouces.

20 Neptune, hauteur 5 pouces, fur un pied de bois doré.

2 I Pluton avecle Cerbere, ce bronze porte $s$ pouces 9 lignes, fur un pied 40 . de marbre blanc, \& Minerve avec fa paterre, de 3 pouces de haut; deux autres petites figures de bronze. 
$\rho 4$

Bronzes.

8. 2. 22 Un joli bulte de Minerve de 5 poures 6 lignes de hauteur.

11. 2.23 Veftale portant une lampe, hauteur 8 pouces 9 lignes.

8 - c $^{24}$ La Déefle Vefta \& un Saturne affis avec fa faulx : ces deux figures portent chacune 2 pouces 3 lignes, $\&$ une Flore de 5 pouces.

g. 22 Bacchus enfant fur une grenade, figure en pied des pouces de haut.

12 - i5. 26 Le bufte de Bacchus dans fa jeunefle, \& celui de Silene bien conditionnés, haureur de chaque 4 pouces.

9 - -27 Un fuivant de Bacchus avec l'Amphore, haureur 7 pouces 9 lignes.

16. $2.27^{*}$ Diane d'Ephefe, d'albâtre, hau. teur 8 pouces 3 lignes, \& la Pucelle d'Orléans en albâtre, jolie figure.

$12-1.28$ Phœbas, figure votive de bronze antique, hauteur de 3 pouces $;$ lignes.

13 - 129 Cinq petits Amours, dont un défolé, un aurre avec la fleche.

12 . 30 L'Ámour portant un globe, figure debout avec trois Dauphins; ce bronze moderne porte 7 pouces 3 lignes de haut.

17 . 10 , I Lucrece, haureur 8 pouces. 
32 Priape, le Dieu Pet, celui de la $\overrightarrow{6}, \overrightarrow{6}$

Pareffe, la Déeffe Mulcea \& Harpocrate avec ungrand panache, tous petits bronzes.

33 Un Faune \& un Triton avec fa conque, hauteur de chaque 6 pou- 30 . ces.

34 Un Satyre, les bras manquent, \& un Faune, hauteur 7 pouces.

35. Un Satyre \& fa femme, bronzes italiens en pendants.

36 Satyre fur une chimere, deux figures de l'Abondance \& la Gourmandife fur un pied de marbre.

$g-12$

$12-1$

37 L'Abondance \& l'Occafion, hatsteur de chaque 5 pouces.

38 Prêtre voilé tenant l'Acerra; autre avec fa paterre, une Prêtreffe auffi avec fa paterre, Mercure, l'Abon. dance, $\&$ un Amour aufi avec l'abondance : ces cinq bronzes font de différentes grandeurs.

39 Mercure avec le cafque de Pallas 11 - L. \& la bourfe de Mercure, hauteur $s$ pouces.

$40 \mathrm{La}$ pareille figure. $\ldots$

g. 2

12. 5 


\section{Bronzes, Ėe.}

60. $54^{\text {I }}$ Le Dieu Canope accroupi \& couvert d'hieroglyphes, de bafalte; ce morceau porte 9 pouces de haut.

6 - 42 Ofiris, les bras \& la coëffure mutilés, hauteur 4 pouces 9 lignes, \& une figure Egyptienne mutilée ayant au menton la racine de perfea, haureur 3 pouces 6 lignes.

$12 \ldots 43$ Un Prêtre Egyptien : ce bronze bien confervé porte 6 pouces de haut.

12 . 44 Autre Prêtre Egyptien, hauteur 6 pouces 6 lignes.

g- (I) 45 Deux Prêtres avec anneau à la tête avec différentes coëffures, hauteur 3 pouces 6 lignes.

3. 3.46 Truis Prêtres, dont un avec anneau fur l'épaule, chacun porte 2 pouces 6 lignes de haut.

4. 8. 47 Quatre Prêtres, dont deux pour fervîr d'amulette.

13 - 48 Prêtre à tête rafe, tenant la me. fure du Nil, hauteur $s$ pouces.

$10 \quad \ldots 49$ Autre Prêtre plus petit tenant aufi la mefure du Nil. 
fo Autre avec la mefure du Nil, hauteur 4 pouces 6 lignes.

s I Prêtre affis déroulant un livre : ce bronze porte \& pouces 6 lignes de haut.

S2. Un autre Prêtre avec quelques différences.

S3 Autre plus petit aufi déroulant un livre.

\$ Bacchus Egyptien, hauteur 3 pouces 3 lignes : certe figure eit très rare.

ss Autre Bacchus Egyprien affis, furmonté d'une double coëffure; l'une avec les cornes \& le difque, l'aurre avec l'épervier \& des fleurs de lotus; hauteur 5 pouce 6 lignes.

s6 Deux petits Prêtres Egyptiens affis; fur des pieds d'albâtre.

S7 Une table Egyptienne gravée à la page 74 des Antiquités du Pere de Montfaucon, tome 3. part. 1. pl. $S$ XLII. Elle eft de pierre $\&$ porte 16 pouces de haut, fur $2 \mathrm{~s}$ de large. $\mathrm{Ce}$ morceau avec figures \& hieroglyphes, paroît moulé d'après un monument Egyprien.

s\& La Déeffe Ifis ayant fon fils Orus $12-14$ $A$ iv 
8 Figures Ėgytiennes.

fur fes genoux: ce bronze porte 7 . pouces 6 lignes de haut.

10. 59 Autre Ifis fans Orus avec un ornement de tête fingulier, hauteur 6 pouces. 60 Deux plus petites figures d'Ifis,
dont une avec Orus.

8 - 6I Ifis avec Orus, ce bronze porte 4 pouces 6 lignes de haut.

18 - 62 J fis \& Anubis avec une légende, fceau d'agathe orientale, \& un anneau antique de criftal de roche.

$3-63$ Ilis, figure en terre cuite, antique de $\sigma$ pouces de haut.

16. 1.64 Ifis affife, bronze doré, avec hieroglyphes fur la bafe, hauteur 10 pouces 6 lignes.

$150-1.65$ Le Bauf Apis, de la plus belle confervation \& antiquité : ce bronze porte 3 pouces de haut, fur $s$ pouces de long.

8- 19 ( 66 Un autre Bœuf Apis plus petit,

67 Aurre encore plus petit d'une fabrique barbare; \& un d'une belle fabrique.

46. 13. 68 Le Dieu Anubis des Egyptiens ayant une tête de chien, les ailles aux pieds, \&c. hauteur 7 pouces. 


\section{Figures Egyptiennes.}

Une autre figure portant le bâton fourchu d'une main, \& de l'autre la croix, c'étoit la marque des crues du Nil; Orus emmailloté portant la girouete à tête de hupe, \&c.

69 Deux très petits Bœufs Apis accroupis, \& une Momie de bronze; une Momie de porcelaine émaillée: en bleu, \& Abraxas, fcarabée de pierre de rouche.

70 Une Momie de porcelaine grife; 12 - 1. hauteur 7 pouces 6 lignes.

7 I Cinq autres auffi de porcelaine. $-J \cdot 4$

72 Cinq ditro.

Bronzes des Indes, de Canada E de la Chine.

73 Trois Divinités Indiennes, une debour, hauteur de 4 pouces 6 li- $21-\mathcal{S}$. gnes, les deux autres alfifes, chacune de 4 pouces: ces bronzes font eftimables dans leur genre.

74 Trois autres affifes, dont deux à - 9 - 1. têtes d'éléphant, un d'eux a une femme fur fes genoux : on croit que: c'eft le Dieu Brama.

75 Deux Divinités femelles, figures 14.2. A. $x$. 
30 Bronzes des Indes है autres.

13- 1. 76 Deux petites Divinités affifes, l'une à tête d'éléphant, l'autre à tête de cheval. Une Divinité femelle en pied, $2 x$ un Confucius, fur pied de bronze.

12 - 77 Cinq petites Divinités toutes différentes.

16. $10.78 \mathrm{U}_{\mathrm{n}}$ Confucius, hauteur 2 pouces 9 lignes, \& deux figures Chinoifes, dont une portant un pot à parfum, hauteur de chaque 4 pouces 6 lig.

g. 11. 79 Deux différentes figures de Jagrenat, Idoles Indiennes : ces bronzes finguliers portent chaque 7 pouces de haur.

y. 10 So Le Sultan Mogol, affis : cetre figure elt $d^{\prime}$ un bon travail, hauteur 6 pouces 6 lignes.

10 - 1. 8I Une Bambochade Chinoife.

82 Une Idole du Canada de pierre

4 . 19. 82 Une Idol

13. 9 $8_{3}$ Une autre Idole en bronze : on prétend que c'eft un Dieu Helvétien; on peut en voir la figure \& la defcription dans le Journal Helvétique de 1760 . Jo. Hoferi flii.

$36 \ldots-84$ Un vafe à deux anfes fur trois pieds compofés de branchages, for 
Bronzes des Indes \& autres. 1 I diametre eft de 6 pouces 6 lignes. Ss Aurre vafe quaré long à deux an- 4 - 11 fes fur quatre pieds, il y a des ornemens gravés fur le corps.

86 Deux autres de différentes formes, dont un avec un nom allemand fur 6 ? la panfe.

86 * Caffolette avec un couvercle repercé \& des figures Chinoifes en bas relief au pourtour, hauteur $613-5$. pouces.

87 Deux vafes à bouches évafées, 86 deux allongés de différens models. $\$ 8$ Quatre petits vafes de bronze en forme de fruits, un cheval \& un taureau fur des foucoupes \& une théiere compolée d'un oifeau de $\boldsymbol{S}$ - ig terre de Boucaro. 8, Un Eléphant fur lequel font un baldaquin \& deux figures en bron- 2 h. 2 ze.

Différentes figures d'hommes, de fermmes, \& autres morceaux, dont plufieurs antiques.

20. Deux femmes, dont une Danfeu$\mathrm{fe}$, en bronze, hauteur de chacune 16.4 \& pouces 6. lignes. 
10. 8 9r Un Luteur \& un Gladiateur, en pendants, hauteur $s$ pouces.

is ... 92 Deux autres figures d'homme, dont un tenant une paterre.

if - 1093 Un monttre emblématique de tous les vices, hauteur 6 pouces, \& une figure.

25. 494 Un Pocillator ivrogne, ayant dans une main une paterre, dans l'autre un vafe à boire ou clochette; un Coureur \& un Sauteur cafqués, \& deux autres figures.

18 - 9.5 Le Dieu du filence, hauteur 7 pouces 6 lignes, \& Pallas fous la figure d'un oifeau, fa tête eft cafquée \& elle porte un bouclier, deux lances lui fortent de derriere le dos: c'étoir l'annonce de quelques expéditions au retour du printemps.

$2 y$ - 96 Trois figures en pieds de différentes fabriques.

8- 19 97 Une Venus mutilée, dans le goût Etrufque, \& un homme portane un panier:

$9-19^{8}$ Un Priape, deux Hercules, dont un avec la peau de lion, fur des pieds d'albâtre; la Déeffe Ifis avec Orus, $\&$ deux autres petites figures.

4. 14 29 Six autres figures. 


\section{Différens Bronzes, Ec.}

100 Cinq petites figures, dont un Pocillator, \& une femme Etrufque. I0 I Deux Préféricules, dont un avec figures en relief.

102 L'Amour fur un dauphin, bien réparé : ce bronze porte 12 pouces, 30. fur un pied de marbre blanc.

$103 T$ rois petits lions $\&$ un chien de bronze; plus un cheval de mar- 12 bre.

104 Deux lézards, un lion \& un bouc de bronze, un dauphin de la tête duquel fort un homme vêtu : ce joli morceau eft de plomb.

ros Amphibene avec fa coëffure de divinité, une tête de bélier d'un 12. 3. beau travail, deux petits cercopitheque $\&$ un finge.

106 Une cafolette indienne fous la forme d'un animal chimérique. 10. 8. 42 4. 2 $16 \cdot 4$

107 Un bufte d'homme d'un beau travail, un fragment de tête $\&$ d'une main en bronze, \& un mafque an tique de marbre.

108 Le bufte de Didon, celui de

Cléoparte, trois petites têtes \& une 


\section{4}

Différens Bronzes.

l'hiftoire de Remus \& Romulus;

en bronze antique, hauteur 2 pouces 2 lignes.

16. L x so Figure d'Empereur en bronze do. ré ; figure Confulaire portant la toge; l'Empereur Tite \& un autre Empereur.

3 - 12.111 Le butte de l'Empereur Galba, en albâtre.

33 -. 112 Deux Impératrices élégamment drapées, en albâtre, dont une repréfentant Cérès; les têtes, les pieds \& les bras font de bronze, l'une eft mutilée des deux bras.

15. $S^{113}$ Un Courtifan du regne de Louis XIII. Une amuletre ithypallique, antiquité Romaine, fur un pied d'albâtre, \& deux autres figures.

24. $S$ I14 Un Druide en fer, une Divinité Gauloife, \& S. Sébaftien de Jayer , en bronze.

24 _ 115 Trois trophées, de chacun 4 pouces 6 lignes de haut; le modele d'une petite armure avec le cafque, \& une couronne antique, le tout de bronze.

13. . $\$ 6$ Deux têtes en marbre, dont une de Jupiter, fur deux buftes de plâtre, 


\section{Différens Bronzes.}

\& une troifiéme en marbre d'un Em. pereur inconnu.

117 Une Sultane Mogole, ou plutốt $\bar{G}$.

un Fétiche ou Idole Barbarefque, de bois, hauteur 14 pouces.

I 8 Deux lampes de bronze, dont une eft une elpece de tête de négre, avec la boache allongée pour fervir de lumignon.

I 9 Ving-neuf lampes de terre cuite que l'on divifera.

ig).

320 Une lampe à trois lumignons en forme de vafe triangulaire fur une tige terminée par un trépied avec 15. 4. ornemens de bon goût, modele Romain de bon goût en bronze.

12 I Aurre lampe avec des ornemens -9 -1. élégans, ouvrage d'Italiè.

12.2 Autre lampe Romaine, compofée d'une efpece de fphinx à trois 14. 1 . pieds.

123 Un enfant debout, qui porte une coquille fur fon dos, hauteur 4 pou- is ces 6 lignes, fur un pied aufi de bronze doré.

324 Un aurre enfant, tenant une moitié de coquilles en bronze.

(


bronze épineux pour les Martyrs; $\&$ deux clochettes antiques.

3y.. 126 Cuiller fervant à une paterre, longueur 5 pouces; deux fibules ou agraffes à l'ufage des femmes Eirufques', \& dix pieces, tant clefs que cadenats.

A5 $-1.126^{*}$ Des fceaux, des bagues \& des cachets.

6. 1. 127 Une urne de pierre pour la fépulture d'un épervier.

3. 12. 28 Une belle urne moderne.

\section{Vafes antiques de verre.}

35 - (1) 129 Un beau vafe à deux anfes bien confervé, hauteur 9 pouces $6 \mathrm{li}$ gnes.

22 - 1. $x 30$ Une bouteille quarée à une anfe; c'eft un morceau recommandable.

45 - I 3 I Une urne cinéraire à côtes avec fon couvercle, haureur 9 pouces.

1 - 10 I32 Autre vafe à deux anfes, done un eft caffé \& le corps fêlé , \& quelques fragmens de verre antique.

32. 6. I33 Quatre lacrymatoires de verre antique, dont l'un a la panfe en. vironnée d'une ligne fpirale d'une. grande beauté. 


\section{Terres antiques, Ec.}

134 Trois vales, trois lacrymatoires,

$\&$ deux petites écuelles de terre an- 6 .

tique.

I3S Dix Fétiches de fauvage, en terre. i2 6 .

136 Deux haches \& un petit piédouche antique de bronze, deux autres haches de jadde \& une de 5 . (9). pierre.

Bas-reliefs, Buftes de marbre,

Terres cuites, Ėc.

137 Un petit tombeau de marbre bien confervé, avec une infcription ot- 69 née de fculprures en relief, hauteur

12 pouces.

138 Modele d'un maufolé en terre avec cuite. "1.

139 Deux infcriptions tumulaires fur? marbre blanc.

140 Le couronnement d'un petit tom- $(S-$ (S) beau à deux frontons, avec des ornemens fymboliques, en marbre blanc, \& deux débris de pavé \& in. cruftation en mofaique antique.

14I Un bas-relief moderne ancien; 6. 4. qui paroît repréfenter un fujet fatyrique.

I42 Un facrifice à Bacchus, bas-re- 48 - 1 
lief de marbre de 8 pouces 6 lignes

$\rho$ de haut, fur 14 de large dans une bordure dorée.

3. $6: 43$ Un Termes, figure d'Hercule -..en marbre blanc, hauteur 19 pous: ces.

4. $18.144:$ Un bufte de femme en relief de marbre plaqué fur ardoife, \& le mafque de cartouche en plâtre.

15. 3.145 Les trois Graces, bas relief de terre ronge, hauteur 8 pouces, lar. geur $s$ pouces, dans une bordure - dorée.

'Ly. L 146 Un facrifice, très joli bas-relief 2. d'argent, hauteur 2 pouces 6 lignes, largeur 9 pouces 6 lignes, dans une bordure rouge.

12 . 147 Deux bas-relief de marbre, l'un repréfente l'Amour \& l'Amitié, l'autre Léda, fur des pieds de bois.

Is - 1. 148 Le bufte d'une Vierge de pisié, - en bas-relief de marbre blanc, de forme ovale, hauteur is pouces, - largeur 8 pouces, par L. Delvaux, dans une bordure noire à filets dorés. - 49 Detix buftes, dont celui d'un
Empereur, en marbre blanc, fur des gaînes de bois peint en marbre. 
Ouvrages en bois \& cire, \&c. 19

I so Le bufte de Jacques Shakefpeare, fameux poéte Anglois, de grandeur 6 . ig naturelle, en plâtre.

I I I Saint François en méditation,

- figure en ivoire d'un bon travail, hauteur 5 pouces, fur un pied de 15.5. bois.

152 Le bufte de Louis XIV \& celui du Dauphin, fon fils, en bas-relief d'ivoire par un artifte diftingué ; ces deux morceaux font de forme ovale, $\&$ chaque porte 4 pouces 3 lignes de haureur, fur 3 pouces 6 lignes, dans des bordures dorées.

193. Plufieurs figures en bois que l'on 13. S. divifera. 2 grounes 2 figuers - $8-2$.

I $\$ 4 \mathrm{Le}$ bufte de Scaron en cire colorée, très de relief, fous verre $\&$ bordure noire.

15s Autre bufte d'homme en cire, par Ifaac Go/fet, célebre artifte An. glois, fous verre \& bordure noire. $J$.

156 Deux enfans, de ronde boffe en cire, dans des bocaux de verre. $11-1$. Figures de pâtes des Indes.

${ }_{157} \mathrm{Un}_{\mathrm{n}}$ vieillard Chinois à tête branlante, hauteur 12 pouces 6 lignes.

19. 12 
20 Figures des Indes.

22. - 58 Deux autres figures, l'une d'hom: me, l'autre de femme habillées d'étoffe, hauteur de chaque I 3 pouces.

12 - 159 Un homme \& un enfant, les têtes \& les mains font branlantes.

10 - 10 160 L'Empereur du Mogol \& fix au. tres petires figures de pâte de pierre de laar $\&$ de porcelaine.

\section{Tableaux Eु Modeles.}

100 . 161 Deux tableaux mouvants, l'un repréfente un Rémouleur, l'autre un Scieur de bois, chacun porte $\mathbf{I}$ pied de haut, fur 8 pouces 6 lignes de large, dans des bordures dorées.

many. $1 \sigma_{2}$ Un tableau très bien peint com. pofé de différens gibiers \& volailles, $\&$ d'un jeune garçon, fur toile qui porte 3 pieds I I pouces de haur, fur 5 pieds 2 pouces de large.

15 - 1.163 Deux tableaux de fleurs fur toile, de chacune 2 pieds 8 pouces de haur, fur 2 pieds I pouce de large.

24. S $16_{4}$ Quatre tableaux peints fur toile, chacun porte 30 pouces de haut, fur 24 de large; ils repréfentent Fernel, 


\section{Médailles \& Monnoies. 2 I}

Bartholin, Paré , \& Dulaurens.

164 * Plufieurs tableaux \& eftampes

- fous verre que l'on détaillera.

165 Une des façades du Louvre en

carton, hauteur 8 pouces, longueur

3 pieds 3 pouces.

$165{ }^{*}$ La maifon d'un Meunier, \& un

autre édifice avec figures mouvan- 66 .

tes par le moyen du fable.

Médailles É Monnoies d'or, d'argent, $\mathcal{E}$ de cuivre.

166 Trois médailles d'or, dont une très grande de l'Impératrice de Ruffie, Catherine II, elles pefent un 890 . marc un once demi-gros.

167 Trois autres médailles, dont une très grande de ladite Impératrice de Ruffie, elles pefent fix onces fix $6 \%$. 2 . 48. $15 \cdot 8$ 


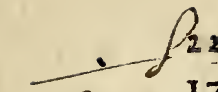

\section{Rig}

51. 1.70 Elifabeth I. \& deux autres médailles en argent de Catherine II, Impératrice de Ruffie, pefant fep: onces quatre gros.

y9. 71 Dix médailles d'argent pefant un marc quatre onces : favoir, Catherine II de Ruffie, l'Empereur Charles VII , l'Empereur François I, le Prince Charles, fon frere, cinq pieces de la Reine d'Hongrie, \& une de Frederic Hoffmann, Médecin du Roi de Pruffe.

22 - ag 172 Cinq pieces de Ruffie \& de l'Empire, en argent, pefant quatre onces un gros.

86 - 1. ${ }^{17} 3$ Une grande médaille de Louis XV, trois de l'Ordre de S. Michel, Geoffroy \& Vanswieten, Médecins, une médaille de la Calotte, deux autres pieces \& fix jettons, le tout d'argent pefant un marc quatre onces deux gros.

10 - 174 Sept médailles ou monnoies de Papes, une médaille fatyrique contre les Papes, \& une perite piece de Jean Hus, en argent, pefant une once trois gros \& demi.

y. 12 175 Seize petites médailles antiques 


\section{Médailles \& Monnoies.}

d'argent, elles pefent une once trois gros \& demi.

I76 Dix neuf perites pieces, dont plufieurs copiques de Ruffie, \& 23 pieces de monnoies d'Allemagne; Ruffie, Turquie \& des Indes, en argent, elles pefent quatre onces cinq gros.

176 * Un pied-fort de Louis XI, \& 3. 10. une monnoie de Sigifmond, Roi de Pologne, en argent.

17 Quinze pieces de différentes monnoies, dont trois piaftres d'argent, is) - S le tout pefe trois onces un gros $\& z$. demi.

178 Trente-fix médailles antiques \& modernes de bronze.

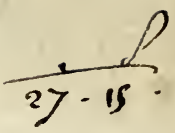

79 Dix médailles de bronze, dont le portrait de Martin Folkes , \& celui de Jean Frind, Médecins cé-g - 13. lebres Anglois, Charles VII, Empereur, \& Stanillas, Roi de Pologne.

180 Soixante - feize pieces de monnoies, jertons \& médailles en cui- 9 - 1 vre, dont plufieurs hiftoriques \& fatyriques; quatorze piecesen étain, \& deux têtes de bas-relief fculprés fur de la pierre à rafoir. 
'Armes, Uftenfiles, Habillemens

-20. L 1.8 I Une arme compofée de deux cornes accouplées enfemble, dont le haut eft garni d'ivoire, \&z deux haches d'acier à manche de bois.

5. L $18_{2}$ Un poignard des Malabares, un autre à quatre faces \& deux caffetêtes.

22 - I83 Trois calle-têtes \& deux autres armes.

4. S. ${ }^{8} 84$ Une arme à l'ufage des Indiens, un arc \& un carquois garni de $\mathrm{fle}$ ches.

8- 19 I 85 Un carquois de fauvage garni de ceintures $\&$ autres agrémens, un 12 .... arc, une pique, \& fix paquets de fleches.

15- 1 I86 Sept paquets de fleches \& une - pique.

8 - I 87 Un arc \& onze fleches. I 88 Un couteau à manche d'agarhe y- 9 dans fon foureau garni de cuivre doré , orné de pierreries; trois autres dans une gaîne d'ivoire, qui repréfente trois poiflons, \& un coureau à deux perites lames. 
Armes \&' Habillemens.

18, Un nécelfaire Indien compofé 10.12 d'une cuiller de métal, d'une fourchetre \& d'un couteau dans un - étui.

a 90 Cinq bonnets, une ceinture, deux tabliers, un carquois garni de fle- 6. 2 . ches, "\& deux bourfes.

19I Un tablier orné, deux boîtes ì poudre, deux bourfes, des brode-17. 4 . quins, deux napes d'écorce d'arbre, \& un bonnet de plume.

92. Une boîte à poudre de corne d'animal des Indes, ornée en ivoire $15 \cdot 3$

\& \& garnie en étain.

193 Sept pairés de fouliers \& pantou- Hes \& autres objets.Indiens \& fau- 15 (9). vages.

9+ Un habir de Mandarin fair de gaze gros bleu, des brodequins, un bon- 130 net, un calumet $\&$ un couteau. 195 Un autre habillement de! Mandarin auff de gaze avec des bro- $\$ 0$. - dequins.

396 Deuxboîtes à poudre, unebourfe,

"Ine pelotte dans fon étui d'ivoire,

zs une tablettè dans fon étui d'écaille, 2 deux autres pieces Chinoiles.

$\$ 27$ Unẻ balánce, une écritoire $\&$ una ig -. - B $\cdots=1$ 
pierre pour broyer l'encre, le tous

$\int$ à l'ufage des Chinois.

21-1. 198 Deux fouets, un peigne, deux calumers, \& autres objets étrangers. 7. 99 Un parapluie \& quatre écrans
chinois.

22 _. 200 Quinze pieces, tant nappes que ferviettes, variées de couleurs, ren. fermées dans une boîte. Ly - 201 Un bonnet chinois \& deux ai-
grettes d'argent, d'un travail riche, \& un petit vafe travaillé en filigrane d'argent.

12. 19, 202 La marche de l'Empereur de la Chine, grande frife; une tablette, un livre \&z deux boîtes avec des fufées, plus deux feuilles de gaze fur lefquelles font peints des oifeaux; fous verre \& bordure.

Ouvrages de laque, ivoire, Ec. 10. 1y. ${ }^{20}$ Trois écrañs de pierre de laar; $22 \ldots 204$ Deux écrans ornés de fleurs en relief d'ivoire, le corps de bois des Indes.

12. 6 2os Un jeu avec des fiches \& jettons d'ivoixe. 
Ouvrages de laque, ivoire, Ec. 27 206 Un platteau \& deux petits pieds, 9- ig d'ancien laque. 207 Une écritoire, une boîte à poudre, un platteau, une foucoupe $\&$ : 8 . un coffre de laque.

208 Deux vafes ornés de branchages,

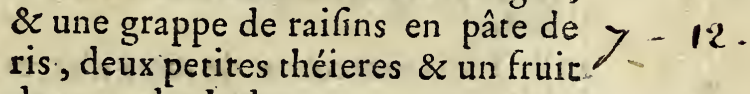
de terre des Indes.

209 Une tour chinoife de porcelaine ou terre verniffée, hauteur 2 pieds. 19 - 1 . 210 Une grande jatte $\&$ fon platteau - en écaille.

2I I Un manche de fabre à l'indienne 16. 4 . de cuivre argenté, un poignard d'acier à lame dorée, un autre de fer, $\&$ une petite corne travaillée.

\section{Minéraux.}

212 Une roche de quartz caverneufe, 8 un autre morceau de mine d'or, dans des quartz du Pérou.

213 Un riche morceau de mine d'ar- $29-1$. gent avec des quartz du Poiofi.

2 I 4 Un autre d'argent en lame dans une matrice de quartz. Ce morceau $4 s^{\circ}$. 1. tient de l'argent vitreux.

215 Un morceau de mine d'argent de 21 - 1$\mathrm{B}$ ij 
même nature que le précédent, \& un d'argent rouge criftallifé.

6. 2. 216 Quatre morceaux de mines d'argent, dont deux en végétration.

3 - 14. 217 Un morceau de mine d'argent rouge, un $d^{\prime}$ argent gris, \&z un de $C_{i-}$ nabre.

8 . 218 Trois morceaux de mine d'argent gris, un de cuivre foyeux, un bleu de montagne, \& un autre de cuivre.

8 - 219 Plomb, galene \& pyrites cuivieufes, en tout neuf morceaux.

3 - 220 Plufieurs morceaux de galene \& pyrites cuivreufes, \& de la mine foyeufe dans un bocal.

8 - 221 Un murceau de galene criftallifée, trois autres d'ćtain, \& un mor. ceau d'ématite.

3 . - 222 Sept morceaux de matiere ferrugineufe.

3 - 223 Quatre morceaux d'ématite.

2. 10224 Un rocher de fpath vitreux cu= cuivreufes. Ce morceau tient un peu de galene; plus cinq pyrites. 
Subftances falines \& inflammables.

225 Un petit damier \& fes dames 10 . d'ambre, garni en ivoire.

226 Un petit coffre \& une tabatiere d'ambre. $21-1$.

227 Un chapelet \& deux colliers d'ambre. $-5 \cdot \%$

228 Trois pommes de cannes, ure caffoletre \& une coquille d'ambre. Y IO .

229 Un petit homme accroupi, d'ainbre, avec des bottines d'argent. 8. 1.

230 Plufieurs morceaux d'ambre \& de fuccins, \& de l'amiante. $16 \cdot 9$. 23 N. S. fur la croix, un petit autel, un chapelet, un flaccon \& deux grouppes de tourterelles : le tour de 4. S. fel gemme.

Criftaux $\mathcal{E}$ criftallifations.

232 Un criftal brun contenant de l'a. miante \& un bloc de criftal d'If-4y-19)lande.

233 Un crittal de Madagafcar, un de 4.4 . roche, \& deux autres morceaux.

234 Sept différens morceaux de criftaux, \& un de prime d'amétifte. $\quad J-1$.

$$
\text { B iij }
$$


30 Stalactites \& incrufations.

mang. 235 Deux petiis morceaux de flos ferif $r i$, \& plufieurs falactites.

5o. 10. 236 Plufieurs incruftations de rofeaur $\&$ autres, dont on compofera des articles.

\section{Pierres fines \& autres montées en bagues.}

Goo1. . 237 Un gros diamantbillant, entouré de dix gros karats, \& de dix petits dans les angles, le tout blanc. Cette bague elt d'une grande conféquen. ce, elle mérite attention.

$262 \ldots 238$ Une émeraude de forme lozange, belle en couleur, entourée de feize menus brillants.

y. 4. 239 Une aurre bague compofée d'une pierre verte de forme ovale imitant l'émeraude, avec deux éclatantes à côté.

200. 3.240 Un faphir d'orient à huit pans entouré de dix-huit brillants jaunes.

2. 4I Une bague de fantaifie repréfen$195 . \quad$ tant un pot de fleurs compolé d'un brillant jaune, de fepe brillants blancs, de cinq faphirs, de trois émeraudes, \& d'un rubis. 


\section{Bagues.}

242 Une turquoife d'ancienne roche,
de forme ovale.

243. Une bague de cuivre comporée de petites vermeilles.

244 Une plume de paon, entourée de feize rubis d'orient.

$2+5$ Une compofition avec arborifation en or, cntourée de feize ropazes de Bohême. 245 Une agare arborifée, orientale 29 entourée de faphirs.

247 Une autre agate orientale arborifée entourée de vingt-rept perits 60 .

brillants

${ }_{24} \mathrm{~S}$ Une jolie agate arborif'é rouge entourée de vingt émeraudes.

249 Un petit fujet antique gravé fur un jacinthe, fon entourage eft compofé de vingt-quatre petits brillants. $9 \cdot 14$ so Un négre en relief fur agate-onyx entourée de quatorze brillants iso. blancs, \& un qui forme le collier.

2 1 Une autreagate-onyx repréfentant un hibou, entouré de topazes de 24 - 1. Saxe \& d'amétiltes.

$22_{2}$ Un mufeau de dogue en relief fur $g-16$ ? une jolie chatoyante.

253 Une onyx en relief, repréfentant 8-19, un bulte d'homme. 
A 2

\section{Bagues.}

12-1 254 Une camée, repréfentant une femme dans un char, tirée par deur lions.

8 - - 255 Un foldat dans le goît Etrufque, gravé en creux fur un beaujade de forme ovale.

c) - 256 Une belle cornaline de vieille roche, gravée en creux, reprélentant une rête d'Empereur.

18 - 257 Une tête de Roi avec fon diadême, gravée fur une belle cornaline, \& montée à jour.

15. 1. $258 \mathrm{Un}$ abraxas ou talifman, gravé fur jade, \& monté à jour en bague tournante.

15. 1.259 Une rête de Seneque, gravée en creux fur jafpe rouge.

s. S. 260 Une bague de cuivre, compolée d'une cornaline, fur la quelle eft gravé en creux une tête d'Hercule.

L. L. . 26I Une bague garnie d'une bouffolle.

Boutons, Cachet Eु Epingle.

1200-1.262 Une paire de boutons compolée de trente-fix brillants montés en or. 16. 10.263 Une autre paire de boutons de manche en cailloux de Rennes, mon: tés en or, 


\section{Agates, Jaspes, \&ic.}

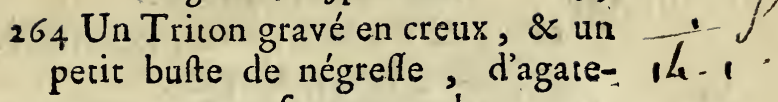
onyx, compofant un cachet. 265 Un diamant taillé en rofe \& mon: 39 té en épingle.

\section{Pierres fines non montées.}

266 Deux opales orientales, une de

Bohême, deux topazes orientales; $12-19$ deux de Bohême, un grenat, un perideau, \& une chryfolite.

267 Deux faphirs, trois vermeilles: 4 . S quatre rubis du Brefil, deux jacintes, deux grenats Syriens, \& une émeraude.

268 Une amétifte, quarre chatoyan: 3. $S$ tes, fix turquoifes, une malaquite, des grenats, \&c.

269 Une petite taffe, un flaccon, qua- 3. S. rante-deux grains, fix olives \& fept autres morceaux de cornaline, plus. des morceaux de turquoife.

$$
\text { Agates, Jaspes } 2 \text { E } a \text {. }
$$

270 . Treizes plaques d'agate. -6. 1.

27x Trente-neuffiches, trente-un jert, 8 - 1. By. 


\section{Agates, Jaspes, E.c.}

tons, \& dix-huit autres pieces triangulaires, le tout d'agate.

$8 \quad-272$ Trente-fept agates, agate onix \& fardoines, dont le plus grand nom. bre a été taillé pour des boutons de manches.

L. 19, 273 Des grains, des olives \& autres morceaux d'agate onyx \& de jafpe, en tout trente fept pieces.

3. $10=274$ Douze olives d'agate-onyx \& de jafpe.

4. L. $274^{*}$ Plufieurs morceaux d'agate \& jafpe

36 . $5: 275$ Une coupe d'agate d'Allemagne. G. 276 Un morceau d'agate rubané \& criftallifé dans fon intérieur, deux plaques de cailloux d'Egypte, \&z deux de Rennes.

80. 3.277 Douze différents petits blocs \& plaques.

9 - 278 Cinq morceaux de lapis, un chapeler de faux lapis, \& une pendelo. que de criftal avec accident.

80. 3. 279 Un petit bloc de lapis.

280 Quatre - vingt-cinq échantillons 4. 1. de marbre d'Italie, de Flandre \& de France : ils font de forme quarée, \&z - portent chaque s pouce 6 lignes. 


\section{Bois pétrifiés.}

281 Deux plaques de bois agatifié polies, \& un morceau agatifié $\& \frac{8}{8}$. criftallifé.

282 Plufieurs morceaux de bois pétrifié

Co $\frac{1}{2}$ uilles follüles É pétrifications.

283 Des vis, des cames \& autres coquilles compofant une petite col- is. 4 lection de folfiles.

284 Des nautiles \& autres pétrifications que l'on détaillẹra.

28 . Deux ourfins pétrifiés, un aure fcié en deux parties agatifées, deux $\boldsymbol{S}$. parties de cornes d'ammon agatifées: $\&$ criftallifées.

286 Plufieurs ourfins $\&$ cornes d'am:mon.

Coquilles univalyes.

$287 \mathrm{La}$ fcalata de 18 lignes de longueur. On connoit la rareté dé cetre: $302-2$ coquille.

288 Onze lepas de différentes efpecess. L . M.

\& une oreille Jans trou.

Bvi: 
11 - 289 Deux nautiles papyracées de la méditerranée, uri gros dauphin, une bouche d'argent, deux fabots Chinois, \& un lepas des inles $\mathrm{Ma}$ louines.

15. L. 290 Deux nautiles épais dépouillés \& $\therefore$ travaillés pour faire voir leur intérieur; il y en a un fur lequel on a. gravé des Aleurs.

6. 4. 29.I Un nautile épais travaillé, un gros fabot poli, un mamelon blanc, \& huit limaçons d'efpeces difié$\rightarrow$ rentes.

10 - 1. ${ }^{292}$ Cinq Jabots différens, un cadran, plufieurs limas \& nerites, en tour ving-fept coquilles \& un operculum.

9. 2. 293 Une unique, plufieurs nerites, une peau de ferpent, \& $\&$ plufieurs autres limaçons, en tout 29 coquilles.

11- (c) 294 Une alêne, une vis de preffoir, une tour de Babel, plufieurs autręs vis, \&x. en tout 22 coquilles.

L. 10295 Une tone nommée perdrix, quatre cafques, dont le tricoté, deur harpes, différentes, une mufique $\&$ un foudre.

6. 2.296 Six casques différens, dont un ftrié, une turbinite, trois mufiques, deux harpes, un foudre $\&$ une tone cancléé 


\section{Coquilles univalves.}

297 Des Cafques, des buccins \& des

tones, en tout 18 coquilles.

298 Une très belle couronne d'Ethispie.

d'un gros volume. -24 .

299 Une plus petite couronne d'Ethio. pie bien confervée, deux prépuces \& trois cafques de deux efpeces. 15. 1 300 Treize coquilles, dont une tulipe; un prépuce, deux buccins triangu- $\boldsymbol{S}-1$ laires.

30I Treize porcelaines, une olive de

Panama, trois buccins ailés, \&e. $3-1$.

21 coquilles.

302 Quinze porcelaines, dont la géographie, plus une olive de Panama. 303 Douze pourpres, dont la tête dैe 4 . (") bécaffe.

304 Deux fcorpions, l'un mâle, l'atitre femelle, une araignée, une chicorée, \& un buccin nommé culotre 10 - 1 de fuiffe.

305 Un fcurpion, un mille - pied's; deux autres araignées, \& une aillée. 1 - 2. 306 Deax belles tulipes en pendans, chacune de 6 pouces 6 lignes. 307 Une brunette, le cierge ou l'onix, un damier, la fauffe aile de papil- lon, le navet, deux écorchées \& deus. olives de Panama. 
$1 \int_{38}$ Cozuilles univalves.

g. 1308 Deux draps d'argent, trois draps d'or, deux fauffes ailes de papilion, une ony $x$, en tout I 4 cornets.

L. Ig 309 Des petites porcelaines \& des olives, dont la litterata, en tout 43 coquilles.

8 . 310 Un buccin terreftre de Cayenne, une veuve, une conque de Triton, \& deux casques à tubercules, dont un travaillé.

7 - 1. 3 I Quatre groffes coquilles qui font deux cafques, dont un couronné, une ailée, \& une conque de Tritons.

- 1 - 3 I2 Trois gros cafques, une ailée, \& une conque de Tritor.

4. 3 I3 Cinq cafques différens \& deux ailées.

8- 1. 3. I4 Trois groffes ailées, un cafque couronné, \& une conque de Triton.

L. 3 Is Deux veuves, dont une dépouillée, un cafque à tubercules, une tonne, une tulipe, \& une porcelains. tigrée:

Coquilles bivalves.

1 - - 3.6 Deux huîtres épineufes, de Sain Domingue garnies de grandes poin: 
tes, dont une adhérente à un madrépore, une petite felle Polonoife, un côté d'une autre efpece de felie Polonoife, qui eft toujours blanche, un petit choux.

317 Une huître épineufe de Saint Do. mingue, deux grouppes d'huîtres de $11-19$ Malthe avec des vermiculaires, $\&$ un choux.

318 Deux huîtres de Malthe, dont une 24 . $S$. attachée fur un teflon, un jambon: épineux avec le biflus, un cceur de bouf voluté, trois cames, dont une polie, une moule fluviatile, \& un grand peigne.

319 Une moule nommée l'oifeau, \& $23-5$. deux cours de Venus.

320 Une rape, une came chagriné qui eft rare, dix tellines, dont plu$38-1$. fieurs rayonnées, \&une moule.

32 I L'écriture Chinoife, un concha is. 4. veneris, une camme applatie peu. commune, \& I 3 autres coquilles. 322 Une moule de Magellan, \& trois. 18. 19. autres moules, dont une fuviatile.

323 Deux valves de moules de Magel- 3 - 1 'lan, dont une fait une rape. 324 Un tiroir rempli de coquilles, $f_{3}-10$ 
40

Ourfins \& Polypiers.

dont on compofera plufieurs articles.

325 Une autre boîte de coquilles.

\section{Ourfins.}

1.. 19. $326 \mathrm{Un}$ ourfin à baguetres, onyx čes grandes Indes, un autre plat percé de fix troux, \& un ourfin viclet en forme d'artichaud.

L. 1. $\left\{\begin{array}{c}327 \text { Plufieurs autres ourfins. } \\ 328 \text { Des étoiles de mer, \& un tête de } \\ \text { Médufe. }\end{array}\right.$ Polypiers.

70. 19, 329 Une belle branche de corail rouge revêtne de fon ćcorce, hauteur Io pouces, largeur 12 pouces.

54. Ig 330 Une groffe branche de corail rouge dépouillée, hauteur \& largeur 6 . pouces. $9-1.33$ I Trois branches de corail fur des.
rochers.

332 Deux branches de corail rouge fur 12 - 1 des pieds; plufieurs morceaux de même corail fur un rocher, \& une branche de mandragore recouvere de corail. 
Animaux, Oifeaux, \&c.

333 Deux branches de corail avec leurs érorces, \& un corail blanc oculé.

334 Une branche de corail oculé jaune de la méditerranée, un madrépore 3.4. bois de cerf, \& un corail blanc oculé.

335 Un madrépore épi de plantain; 12 - 1 . un autre en feuille de choux \& un millepores.

336 Plufieurs madrépores que l'on détaillera.

337 Un cerveau marin, une méandrite

\& deux champignons de mer.

338 Des litophytes \& panaches de mer, dont on compolera plufieurs arti- 1. 4.
cles.

339 Six éponges d'efpeces différentes. L. L. 340 Un grouppe d'éponges à tuyaux \& plufieurs autres.

Animaux, Oifeaux \& Infectes.

34I Deux poiffons coffre, trois poif-

fons armés, le dauphin, un lézard, 6.1 . un ferpent, deux mâchoires de raye, $\&$ une défenfe du poiffon fcie.

342 Un poifon armé, deux petites - 8. 2. tortues, une peau de ferpent, \&c. 343 Un petit crocodille, un lézard \& différens poiffons. 
42 Animaux, Oifeaux, Ec:

is - 344 Six poiffons différens \& une tortue.

G. 10.345 Un crabe des Moluques.

$600-1.346$ Soixantefix poiffons deffechés, tous étiquetés, renfermés dans fix bordures dorées \& fous verre.

18. L 347 Trois aurrescafes à bordures peintes, renfermant fous verre des poiffons aufi defléchés.

y- $\int \cdot 348$ Des ferpens, des lézards dans une liqueur confervarive, refermés dans fix bocaux.

88-19 349 Soixante-fept petites cafes de ver re, contenants des papillons étrangers.

$30 \cdot 10.350$ Quatre-vingtsautres petites cales renfermant des papillons que l'on trouve en France.

3S. 35I Des infectes de différens pays dans cinquante-fept cafes de verre.

$$
\text { Oifeaux. }
$$

$y y$ - 352 Un coq de roche, dans une cafe de verre.

20 - 1. 35.3 Un manucodiata nommé oifeaus de paradis, auffi dans une cafe de verre.

$18 \cdot 2354$ Une cafe de verre dans laquelle 


\section{Oifeaux.}

font une perdrix rouge, un lorior, un merle, un chardoneret, un bruant, un bouvreuil, un ferin, \& une fauvette à têre noire.

355 Un oifeau mouche dans un bocal de verre.

iso Une mélange, un ferio, un petit vanau, us tolla d'eat, un rafle de genêt, un piquebois, \& un lorior.

357 Une perdrix, une bartavelle, la huppe, le coq des bruyeres, une corneille, une pie, une beccaffine.

$35^{8}$ Un émouchet, une beccafte, une bufe, un faifand, un boeland, \& . 9- 10 . un geai.

359 Quatre oifeaux de proie, deux chouettes, \& une corneille mantelée.

he $t$.

360 Un perroquet, un pic-vert, \& un moineau.

36 I Un perroquet gris, un pic-vert; \& un chardoneret. $3-1$. 362 Un pic-vert, un merle à collies blanc, un fanfoner, \& un canard. 7 . \$63 Une perdrix blanche, un bréant; - un pierro fur une belette, un fanfo- y - 10. net, \& deux beccaflines. 364 Un bouvreuil, une bergeronerte, un martin pêcheur, un proulier, 


\section{4 \\ Quadrupedes.'}

une fauvetre à tête noire \& une al-

$S$ louette.

24 - I 365 Un faifand doré mâle de la Chine. $y-4366$ Un toụcan, un pigeon étran$6-367$ Deux becs-à-cifeaux, une jadelle, une corneille mantelée, \& une pie. 6 - 1.368 Lebutor mâle \& fa femelle. 369 Le paon couronné, \& un grand aigle noir.

7 - 370 Jean le blanc, un courli, un heron, \& un autre oifeau.

21 - 10.371 Deux œufs d'autruches pofés fur des pieds de bois, plufieurs aurres cufs.

\section{Quadrupedes.}

6. $4 . \$ 72$ Un écureuil, une hermine blanche, un petit chien, \& une fouris.

15. 273 Un petit tigre ou chat des Indes,.. $\&$ un acouti.

40 .. 374 Un maki dans une cafe de verre pofée fur un pied de bois noirci. if - 375 Un tatou fur un pied de bois. 376 Un tatou \& une tête de requin ? 9-1. portant deux cents quatre - vingt dents.

2 L . 2. 377 Une tête de vache marine, \& une corne en ivoire, dont on a compolé 
Cabinet Anatomique.

un infrument à vent en forme de trompe garnie en cuivre.

378 Deux belles cornes d'animal étranger, elles font polies \& portent chaque 2 pieds 7 pouces de longueur.

379 La repréfentation de deux bœufs.

en carton \& recouverts de peau : ouvrage d'Allemagne. 380 Un taureau \& un cheval. idem. $-\boldsymbol{f}-2$.

\section{CABINET ANATOMIQUE.}

\section{Oftéologie humaine.}

381 Un fqueletre de fotus entier. - - 1.

$382 \mathrm{Un}$ autre fquelette de foetus au: quel il manque une extrémité inférieure.

${ }_{3} 83$ Un fquelette de fœtus boffu, au: quel il manque la tête \& une extrémité inférieure, \& deux boîtes qui renferment des os de fotus féparés. 384 Un fquelette d'enfant aves fes ligamens.

-385 Un fqueletre d'un fujet fait, monté en fils d'archal, pofé fur un pied) d'eftal.

386 Deux thorax fans fernum. - $7 y^{2}-1$. 387 Deux thorax avec leur fternum - $3-16$ ? 
1388 Un tronc avec le baffin.

38 , Un tronc fans baffin.

390 Deux extémités inférieures d'adulte, dont une fans rotule, \& deux estrémités inférieures avec leurs ligamens, dont le fémúr eft fcié vers la moitié.

\section{Oftéologie monftrueuse.}

is. - 39 I Deux fquelertes de fortus féparés dans toutes leurs parties, excepté par leurs têtes qui ne forment qu'une face pour les deux.

18. 10 392 Deux fartus defléchés réunis par le tronc, portant deux têtes, \& les extrémités inférieures de chaque forus féparées.

393 Un fquelette de fœrus qui n'a qu'une feule extrémité inférieure.

394 Deux pieds à fix doigts avec le modele en cire.

395 Une main à fix doigts.

396 Une autre main à fix doigts, dont le doigt furnuméraire eft articulé

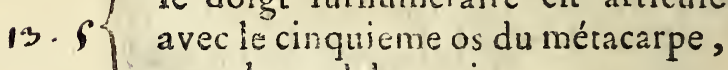
avec le modele en cire.

397 Une autre main à fix doigts, dont le fixieme compofé de deux pha- 


\section{Cabine: anatomique.}

langes, eft articulé par la feconde, avec une apophyfe qui tient lieu de troifieme phalange, \&z qui naît obliquement d'avant en arriere du cinquieme os du métacarpe.

398 Une tête humaine, \&.c. -

399 Têtes humaines de différens âges. 400 Différeniès coupes, verticales de la tête.

401 Cranes, \& bafes de crane d'adulte.

402 Différentes portions de têtes, relatives au trépan, à la carie \& à l'e-) xoltofe.

403 Deux têtes d'hydrocephales, dont 40 .

une entiere.

404 Une têre en ivoire.
405 Une tête en albâtre.

406 Squeletres de quadrupedes.? ... sh . 1

407 Squelettes doifeaux. 408 Squelettes de poifions.

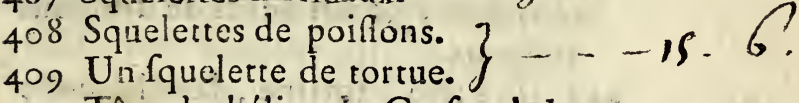

4 ro Tête de bélier de Gerfey à dou-
bles cornes.

4I I Deux cornes de bouquetin.
4 I 2 Bois de daim.

4 I 3 Plufieurs vertebres de jeunes \&

vieilles baleines.
44 Deux côtes de baleine. $\ldots$ 


\section{is \\ Cabinetratomique.}

415 Portion d'os d'une extrémité de baleine ou d'éléphant.

$3 \quad 416$ Deux portions confidérables d'épiderme d'éléphant.

4. 417 Tère de veaul monftrueufe. aur $4 h q-h$

12 - 10.418 Exoftofe de la nature de l'ivoire envoyée à M. Morand par M. Sloane, pour ce que les Naturaliftes appel. lent lacrima elephanti.

Une autre excroiffance offeufe, auffi de la nature de l'ivoire, de la groffeur de la tête d'un enfant nouveau né.

2le = 12.419. Têtes, cornes \& bois de différens animaux, entre autre une tête de fanglier \& une tête de groffe tortue.

2 - 1. 420 Deux boîtes contenant une colleetion de dents humaines de différens âges, '\& des dents de différens animaux.

S6 - 10. 42 I Une tête humaine, dans laquelle au moyen des coupes, on voit routes les cavirés intérieures des différens ós de la tête. 


\section{Pieces anatomiques injectées}

$$
\text { Es préparées. }
$$

422 Un fujet de moyenne taille avoc 43 .

fes mufcles; fes vaiffeaux \& fes nerfs.

423. Un bras humain avec les múfcles

\& lesnerfs. aver 431 .

424 Une myologie de finge, avecles nerfs de la queue, la tête manque. 2 :

42 . Deux mafques de têtes humaines injectés.

426 Un mafque de fortus injecté. .- ?

4:7 Portion de la face d'un enfant injectée.

428 Un péricrane injecté.

429 Une dure mere injectée avec la faulx.

430 Une autre dure-mere, dont les

vaiffeaux font injectés en partie.

431. Un œil humain injecté avec fes mufcles.

432 Un cœur humain injecté. -.. ?

433 Une trachée artere injectée.

434 Un eftomac de fotus injecté.

435 Plufieurs portions d'inteftins in- $3-4$ jectées.

436 Un inteftin crecum injecté. Autro

cœcum préparé. 
so Cabiner anatomique.

437 Deux reins injectés \& ouverts dans leur longueur.

438 Les vaifleaux artériels d'un rein, dépouillés de leur parenchyme, fous verre.

439. Les vaiffeaux artériels \& veineux d'un rein, injectés : les calices du baffinet font en cire.

\440 Une rate injectée \& foufflée. 44I Un cordon des vaifleaux fpermatiques, avec le tefticule, injectés.

3. 4. 442 Un bras injecté, où fe trouvent feulement les arteres \& les veines. 443 Un baffin de fortus avec une portion de l'aorte, \& les arteres hypogaftriques, injectées.

( $\$ 44$ Un baffín d'enfant, où fe trouvent une portion de l'aorte, les arteres iliaques \& leurs ramifications dans le baffin, avec une portion des arteres crurales, injectées.

445 Faulx humaine, offifiée dans une

8-7.\{ $\begin{aligned} & \text { partie. } \\ & 446 \text { Un larynx \& pharynx humain, }\end{aligned}$ avec leurs mulcles \& la langue, préparés.

A47 Un larynx d'animal, avec tous fes cartilages \& membranes prés parés, 


\section{Cabinet anatomique.}

SI

$448 \mathrm{Un}$ cœur de fotus foufflé.

449 Une véficule du fiel avec le conduit choledoque.

Une véficule du fiel avec le conduit choledoque, \& une partie de l'inteltin duodenum.

Une véficule du fiel remplie de calculs.

Plufieurs pierres biliaires.

450 Une boîte contenant plufieurs membres virils foufflés \& injectés, avec des portions de verge de cheval.

-45 I Une touffe de cheveux mêlés par la plique, ou le kolton des Polonois.

Une touffe de cheveux, \& une dent trouvés dans un ovaire.

452 Plufieurs trachées-arteres de différens animaux.

453 Une verge de baleine de 7 pieds . 6 . delong. wee his.

454. Un ver folitaire dans de l'eau devie.

455 Préparations de l'os temporal, for-? mant une fuite de cinquante $\&$ une pieces, pour fervir à la démonfration de l'organe de l'ouie, \& huic $\mathrm{C}$ ij 


\section{S2 Cabinet anatomique.}

autres pieces de l'os temporal d'un veau.

Un os trouvé dans la tête d'un négre.

456. Kyfte volumineux réfultant de la

* membrane fervant d'envelope à un ovaire, affecté d'hydropifie.

\section{Pieces anatomiques en cire.}

26 ... 457 Modele en cire du qquelette frais de la femme Supiot, morte à Paris en 1752 , dont l'hiftoire a été donnée d'abord à la Faculté de Medecine de Paris, dans différentes affemblées du prima menfis de la même année, par M. Morand le Médecin ( $\mathrm{I}), \&$ dont les détails ofréologiques ont été communiqués par feu M. Morand pere, en l'an. née 1753 , à l'Académie des Sciences, où le fquelette en eft confervé.

(1) Imprimée chez la veuve Quillau, rue Galande, fous le titre Hiftoite de la maladie finguliere, \& de l'examen du cadavre d'une femme devenue en peu de tems toute contrefaite pàr un ramolifement général -des os, \& $c_{9}$ 
$45^{8}$ Coupe verticale d'une tête humaine jufqu'au commencement du thorax, dans laquelle les vaifleaux 12 . font repréfentés injectés.

459 Autre coupe verticale de la tête, dans laquelle les vaiffeaux de la $>0-10$. faulx \& ceux du plexus choroïde font repréfentés injectés.

460 Tête \& col fur lefquels on a re- - ig

préfenté la myologie \& l'angiolo-

gie extéricure de ces parties.

$46 \mathrm{I}$ Tne tête, dont le crane \& les

hémifpheres du cerveau fe démon- 2 l. 1 . tent.

462 Un cerveau avec l'origine des nerfs.

$4_{6}^{6} 3$ Un cerveau compofé de plufieurs pieces, pour en faire voir les diffé-
rentes membranes \& les cavités 24. 16 principales.

${ }_{464}$ Un ceil artificiel, où les paupieres, la fclerotique, la choroide,

la rétine, le nerf optique, l'iris \& le criftallin font repréfentés en grand.

465 Une coupe vercicale de l'œil, où 3o · 10.

les membranes tranfparentes \& les $y-9$ humeurs font imitées en criftal. 466 Os temporal d'une groffeur beau- - is - 1. $\mathrm{C}$ iij 


\section{Cabinet anatomique.}

coup au deffus de la nature; où l'on voit aifément la membrane du tympan, les offelets de l'ouie, le vefti. bule, le labyrinthe, les canaux demi-circulaires, le limaçon \& les cordons du nerf auditif dans leurs pofitions naturelles. Toutes ces pieces fe démontent.

S - 2467 Un autre os temporal de grandeur naturelle où l'on voir les mêmes parties en place; les canaux demi circulaires, \& le limaçon, font ousverts.

6. Ц4 469 Un cour injecté.

470. La repréfentation de l'épine du

g- 12. dos avec une portion des côtes, où l'on voir le tronc de l'aorte defcendante, une portion de la veine cave defcendante, la veine azigos, le réfervoir de Pequet, le canal thorachique \& fes ouvertures dans la veine fouclaviere gauche.

( 4 I Une piece qui repréfente les organes internes \& externes de la génération de la femme, diffequés, avec les arteres \& les veines qui s'y. diftribuent depuis les reins, qui font auffi figurés dans la même piece, avec les uréteres \& la veffie. 
472 Une autre piece qui repréfente les organes de la génération de l'homme, diflequés \& injectés audeffus des reins, qui font auffi figurés, l'un avec fa membrane cellulaire \& ouvert dans fa longueur, \& l'autre dépouillé de cetre membrane.

473 Une portion de l'inteftin rectum avec fon fphincter \& fes mufcles re-) leveurs.

474 Un tronc repréfentant les mufcles droits, obliques, pyramidaux, le cremafter, les glandes inguinales, \& quelques mufcles du thorax diffequés.

475 Une piece repréfentant les parries externes de la génération d'une hermaphrodite.

476 Une piece repréfentant une matrice dans l'état de groffelfe, ren. 1o- L. fermant le fœetus avec fes membra$18 \cdot 18$ nes \& dépendances.

477. Une piece repréfentant les tendons fléchiffeurs \& extenfeurs des doigts de la main avec les mufcles interofleux diflequés.

Une piece repréfentant les tendons extenleurs" \& Héchilfeurs des 


\section{Cabinet anatomique.}

orteils, avec les mufcles interor. feux diffequés.

S - -478 Une piece repréfentant la membrane mufculeufe de la veffie de l'homme, avec les véficules féminales, la proftate \& une partie des canaux déférens.

Une autre veffie avec la proprate.

Une veffie repréfentant fon f phincter \& les troncs artériels \& veineiı

qui s'y diftribuent.

Une coupe de veffie repréfentant une portion des canaux déférens, les véficules féminales, la proftate, \& l'orifice des ureteres dans la câvité de la veffie.

4. 1. $479 \mathrm{Le}$ modele d'un pied à huit doigts.

Pieces en ivoire, Ec.

avee 480 Deux globes, repréfentant l'un $465 \quad$ le nerf optique, la fclérotique \& liris; l'autre le nerf optique, la fclérotique, la choroide \& l'iris, portés fur des fupports d'ambre jaune.

48.1 Un autre globe repréfentant le 


\section{Cabinet anatomique.}

nerf optique \& la fclérotique fans cornée.

842 Un œil artificiel compofé de différentes pieces qui fe féparent les unes des aurres, lefquelles repréfentent ies membranes \& les hameurs de l'œil ; le tout contenu daus un étui.

483 Un autre œil artificiel reprélentant les mêmes parties, contenues 40 pareillement dans un étui. 484 Une oreille avec les offelets de l'ouie en cire.

\section{Pieces en bois.}

485 Deux globes, repréfentants Pun la figure de l'ceil dans.fon orbite ; \& l'autre la coupe tranverfale des 7 - 5 .
membranes \& des cavités.

\section{Pieces en cryftal:}

486. Un globe repréfentant le fclétotique, la cornée, l'iris \& la pu- $S$. pille.

$4^{87}$ Suite d'yeux de verce au nombre so $\cdot 1$.

C $\mathrm{V}$ 
de trente, repréfentant différentes: maladies. Savoir:

No. . . Eil dans fon état naturel, pour fervis: de terme de comparaifon.

2, $3 \& 4$. Ophtalmie dans fes trois premiers: temps.

8. Ophta!mie portée à fon plus haut degré \& accompagnée de chemofis.

6. Epanchement de fang, dans la chambre antérieure. Sugiliatio. Hypofphagma.

7. Cornée empatée, \& parfemée de vaiffeauz limphatiques. devenus fanguins \& variqueux.

8. Onglet. Pterygion.

9. Cornée détruite par un onglet ou farcome: qui en occupe toute 'l'érendue.

ro. Cornée affectée de leucoma ou albugo.

I. Eil échymofé.

$12,13,14,15,16,17,18,19 \& 20$. Diverfes efpeces de cataracties fimples ou ba:rées.

2x. Eil affecté de glaucome.

22. Eil qui a été opéré pour l'extraction du: cryftallin fur la cornée duquel on voit. une cicatrice étroite, demi ciculaire, \& très: apparente, qui décele la trace de l'inftrument, \& où l'adhérence dè l'uvée: à làz plaie faite à la cornée, a caufé un dérangement dans la fituation de la prunelle.

23. Cryftallin paffé dans la chambre antérieure dé l'humeur aqueufe.

24. Abcès dans l'uvée.

25. Prunelle rétrécie, Phrifis pupilla. 


\section{Cabinet anatomique.}

26. Déchirement de l'uvée, qui a occafionné un alongement de la prunelle dans la direction du grand au petit angle. Prunelle de chat horizontale

27. Prunelle dilatée.

28. Eil affecté de goutte fereine parfaire; amaurofis.

29. Eil dont Piris repréfente en quelque forte: un cadran de montre.

30. Cil naturel, mais dont l'iris eft fi noire; qu'on ne diftingue aucunement la prunelle.

4:88 Une armoire qui renferme un très: grand nombre d'os humains; affec-

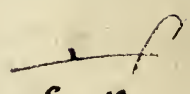

$95-10$ tés les uns d'anchylofes, les autres d'exoltoles, de carie : dés os fractarés \& foudés par le cal; des offifica tions; des exfoliations dont la defeription auroit été trop longue pour un catalogue : on fe contentera feulement de défigner क

Io. Une mấchoire inférieure dont toưte la: bafe eft occupée par une exoftufe plus groffe: que la tête d'un enfant.

20. Une ex oftofe de la partie inférieure du fé:mur, de la groffeur d'une tête humaine.

$3^{\circ}$. Une exoftofe avec carie de la.partie fupérieure du tibia \& du péroné.

$4^{\circ}$. Un fémur exoftofé dans fa partie moyenne: $\&$ inférieure, avec carie dans lintérieur \& des excroiffances offeufes à l'extérieur.

C. v.j. 
$5^{\bullet}$. Une exoftofe de la groffeur du poing far une portion de crâne d'une épaiffeur prodigieufe, \& qui a la même forme, que l'on croît être crâne humain , à raifon d'une couronne de trépan qui y a été appliquée.

60 . Deux grands panniers remplis de diverfes maladies des os, comme fractures, en$\gamma^{k y l o f e s, ~ e x o f t o f e s . ~}$

$48 \cdot 14^{8} 9$ Pierres de reins, de veflies, ou formées dans des rumeurs. Et antres concrétions, incruftations maladives. Savoir :

Une pierre formée fous la langue.

Une formée dans une tumeur.

Quatre groffes pierres tirées en Avril 1734 aux Invalides, par la méthode du grand appareil, à M. de Saint-Michel, guéri de l'opération.

Dix pierres de la groffeur d'une noifette, de nature gypfeufe, tirées de l'urethre d'un enfant de trois ans.

Bout de fonde de plomb perdue dans la veffie, garnie d'une incruftation pierreufe, faifant le fujet d'une obfervation inférée dans les Mémoires de l'A cadémie de Chirurgie, tome 3.

Tente de linge de la groffeur \& de la longuear du petir doigt, recouverte d'une croûte gra-

veleufe en féjournant dans la veffie. Piece donnée par feu M. Collor, Lithotomifte, à feu M. Winnow, qui l'avoit donné à $M$. Morand. Voyez le tome troifeme des Mt-

- moires de l'Académic de Chirurgie. 
Aiguille à tête qui a fervi de bafe à une concrétion pierreufe de grand volume, en $\mathrm{Ce}^{\mathrm{e}}$ journant dans la veffie d'une fille, à Parme. Piece gravée dans les Mémoires de l'Académie de Chirurgié, tome 3.

pierre diune hare.

Modele en cire de la groffeur \& de la forme d'une pierre de 28 onces pefant, portée dans la veffie par $M$. de Poitiers, qui ne s'étoit jamais plaint, \& qui mourut d'apoplexie à $60 \&$ queiques années.

Modele en cire d'une pierre tirée d'un cadavre à l'hôpiral de la Charité de Paris, \&z qui pefoit trois onces, ayant fix pouces 6 lignes de hauteur ', \& un pied de circonférence.

Pierre ftercorale extraite par feu M. Maréchal, ayant deux onces deux gros \& demi de poids, gravée dans les Mémoires de l'Académie de Chirurgie, tome troifieme.

Concrétion très finguliere \& fort curieufe formée dans le canal inteftinale d'une femme, laquelle avaloit des os de pied de mouton, que l'on reconnoît pour avoir fervi de bafe à cette concrétion. L'obfervation en a été publiée.

\section{Pieces de Méchanique.}

490 Un beau télefcope d'environ 30. pouces.

491 Un microlcope garni de toures - 24

fes pieces.

492 Unautre microlcope. $\ldots \ldots$ 
$\int G_{2}$ Pieces de Méchanique E Bijoux.

3- 1493 Un autre microlcope d'une conftruction différente.

25 1.494 Un petit microfcope Anglois dans fa boîte de chagrin.

y - " $494^{*}$ Deux petits microfcropes.

18 - 495 Une lunette d'approche faite par Sayde, Opticien du Roi.

14. 1. 496 Un cilindre d'optique avec treize cartons peints.

2h - 1 - 497 Une machine pneumatique.

12 . $498 \mathrm{Un}$ miroir de métail, convexe d'un côté, \& concave de l'autre, monté fur un pied; fon diametre - eft de 7 pouces 6 lignes.

10 - 13.499 Un oprique.

3 . 19.500 Un optique en boule.

9 .. Sor Une boule de verre étamée. 9 .

5. 2.503 Une lanterne magique. $\overrightarrow{9}$.

4. 1. $\mathrm{SO}_{4} \mathrm{Un}$ aimant artificiel.

$$
\begin{array}{r}
\text { so4 Un aimant artific } \\
\therefore \quad \text { Bijoux. }
\end{array}
$$

88 - 505 Une grande croix de l'ordre de Saint Michel en or émaillé, pefant i une once demi-gros vingt-quatre grains.

36. I. jo6 Une plus petite croix de Sains 
Michel en or émaillé, elle pefe quatre gros.

S07 Une autre croix pefant un gros \& 20 . demi.

508 Une boîte quarré-long très bien cifelée, avec le portrait de l'Infante de Parme; on l'eftime à fept onces d'or.

Sog Une boîte quarrée de belle agate d'Allemagne, montée en or travaillé à jour ; elle eft ornée d'infec- 300 . tes compofés d'agate, de fardoine, onix, coraline, jafpe, \&c. Cette piece, très curieufe, eft faite pas Hoffman.

glo Une boîte à cuvette de beau jalpe, garnie d'une gorge d'or émail- 182 . lée.

SiI Une autre boîte en cage de far. doine d'Allemagne, montée en fi. milor.

gi 2 Une boîte de cailloux, montée. 10 . 10. de même.

S13 Une boîte de prime de grenat $150-1$. en cuvette à gorge d'or.

S14. Un petit coffre d'agate d'Allema. 6.12 gne garni en argent.

Sis Une montre à répétition de Juse- 622 
64 Bijoux.

lien le Roi, ornée d'un bouton de diamant, la boîte, les aiguilles \& la chaine font d'or; il y a un cachet, une bouffole \& une lanterne.

162 S16. Une autre montre ordinaire par Thuret, la boîte \& la chaîne d'or.

46 _ $\$ 17$ Une paire de boucles de foulier, $\&$ une de jarretiere en topazes de Saxe.

12. 3.518 Une paire de boucle d'argent à chappes d'acier.

$6 . \begin{cases}519 & \text { Un porre-col d'argent } \\ 520 & \text { Une boucle de col d'argent. }\end{cases}$

g... S2I Un couteau ployant, à manche de nacre de perle garni en argent.

$\$$ - $\$ 22$ Un autre couteau à deux lames, l'une d'argent, l'autre d'acier, à manche de nacre de perle garni en argent.

32 - $-S_{3} \mathrm{U}_{\mathrm{n}}$ couteau à reflort en écaille garni d'or.

y - 10.524 Dix-huit boutons de cryftau montés en nacre de perle.

s2s Des fondes \& autres infruments de Chirurgie en argent.

$y^{2}-10.526$ Un médailler de bois des Indes compolé de dix grands tiroirs \& de quarante.deux petits; il porte trois 


\section{Effets.}

pieds trois pouces de haut, deux pieds un pouce de large, \& quinze pouces de profondeur.

$\$ 27$ Un plus petit médailler en marqueterie compofé de douze tirôirs, hauteur douze pouces, largeur onze pouces.

\$28 Un coquiller à pieds de biches garni de tiroirs \& de deux portes batantes fervant à renfermer les coquilles, longueur fept pieds trois pouces, largeur trois pieds fix pouces.

\$29 Un petit modele d'armoire à deux battants avec des tiroirs en écaille. 530 Un autre de bois noirci orné de figures $\&$ autres agréments incruftés en ivoire.

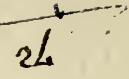

$30^{*}$ Un modele d'armoire pour fervir de coquiller, cumpofé de 17 grands tiroirs, \& de onze pétits dans un fonds, refermé par deux $18-1$.

g. 2 battants; le tout en bois rapporté très orné, \& ayant 4 pieds \& de. mi de largeur, 3 pieds 4 pouces de hauteur, \& $\&$ un pied $\&$ demi de profondeur, porté fur un bâti de chêne de 28 pouces de haut.

S3I Deux petits modeles de chariots 
9. 1 pour tranfporter des malades, \&zautres de machines Chirurgicales.

3 . 19 $\$ 32$ Deux rochers, fur chacun defquels font quatre oifeaux de bois doré.

16. $5 \$ 33$ Deux poiffons faits de nacre de de perle \& petits grains; ouvrage de Robertday.

g. - 534 Deux petits buftes en nacre, ornés de turquoifes $\&$ autres pierres, les têtes font d'émail.

g. 1.535 Les différentes progreffions du ver à foie, fous verre \& bordure.

6 - 1.536 Un bouquet de fleurs dans un vafe, ouvrage en ivoire.

3). 537 Une boureille \& une éguiere de fayence de Morviedro en Efpagne.

$\mathrm{l}_{3} 8 \mathrm{Un}$ coco des moluques. So .. 509 Un herbier en huit porte-feuil-
les. 
Lu \& approuvé le 27 Octobre 1773. MARIN.

Vu l'approbation, permis d'imprimer, ce 27 OAtobre I773. DE SARTINE.

De l'Imprimeric de Didor, rue Pavée, 1773 : 
$\therefore+2+1=0$

4.

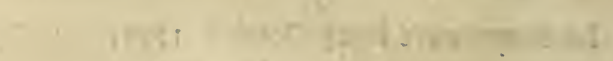
$\ldots 1 \div \ldots$

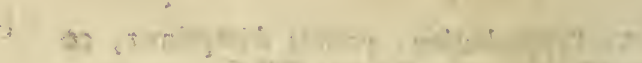

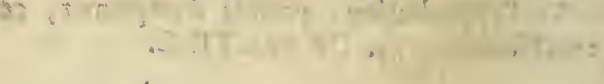

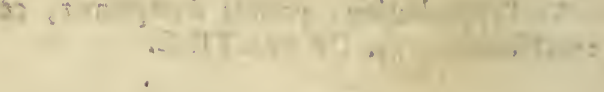

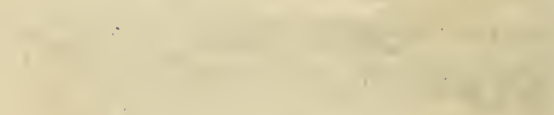

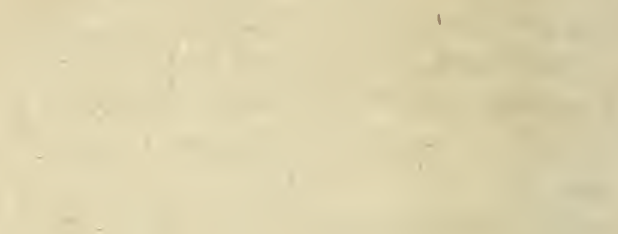

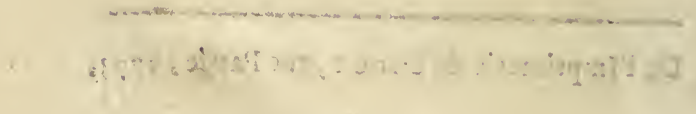

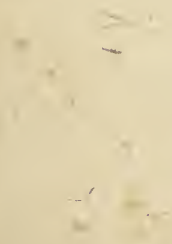







a

$-523) \times(c(c$ $a^{2}+1(12) b y$

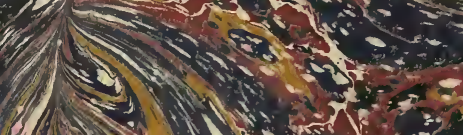

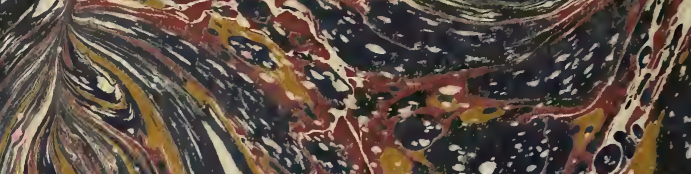
$(1+2)$ (2)

$-1+100$ e. ? t poser

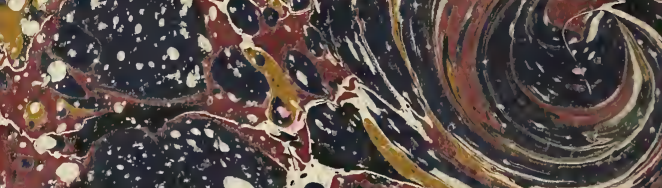
$0-5-5-1-1=$

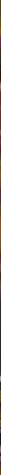

